\section{REFERENCES}

[1] H. K. Gummel and H. C. Poon, "An integral charge control model of bipolar transistors," Bell Syst. Tech. J., vol. 49, p. 827, 1970.

[2] L. W. Nagel, "SPICE2: A computer program to simulate semiconductor circuits," Electron. Res. Lab., Univ. Calif., Berkeley, Memo. ERL-M50, May 1975.

[3] D. E. Thomas and J. L. Moll, "Junction transistor short-circuit current gain and phase determination," Proc. IRE, vol. 46, no. 6 , pp. 1177-1184, June 1958.

[4] M. K. Chen, F. A. Lindholm, and B. S. Wu, "Comparison and extension of recent one-dimensional transistor models," IEEE Trans. Electron Devices, vol. 35, pp. 1096-1106, June 1988.

[5] M. K. Chen, F. A. Lindholm, and T. W. Jung, "Non-quasi-static small-signal models for semiconductor junction diodes with extensions for transistors," Solid-State Electron., vol. 30, no. 8, pp. 883-885, 1987 .

[6] H. Klose and A. W. Wieder, "The transient integral charge control relation-A novel formulation of the currents in a bipolar transistor," IEEE Trans. Electron Devices, vol. ED-34, no. 5, pp. 1090-1099, May 1987.

[7] J. G. Fossum and S. Veeraraghavan, "Partitioned-charge-based modeling of bipolar transistors for non-quasi-static circuit simulation," IEEE Electron Device Lett., vol. EDL-7, pp. 652-654, Dec. 1986.

[8] B. S. Wu and F. A. Lindholm, "One-dimensional non-quasistatic models for arbitrarily and heavily doped quasi-neutral layers in bipolar transistors," IEEE Trans. Electron Devices, vol. 36, no. 4, pp. 727-737, Apr. 1989.

[9] J. A. Seitchik, A. Chatterjee, and P. Yang, "An accurate bipolar model for large-signal transient and ac application," in IEDM Tech. Dig., 1987, pp. 244-247.

[10] J. E. Schutt-Aine, "Determination of a small-signal model for ion-implanted microwave transistors," IEEE Trans. Electron Devices, vol. ED-30, no. 7, pp. 750-758, July 1983.

[11] R. G. Gough, "High-frequency transistor modeling for circuit simulation," IEEE J. Solid-State Circuits, vol. SC-17, no. 4, pp. 666-670, Aug. 1982.

[12] A. B. Macnee and R. J. Talsky, "High-frequency transistor model for circuit design," IEEE J. Solid-State Circuits, vol. SC-6, pp. 320-322, Aug. 1972.

[13] R. I. Ollins and S. J. Ratner, "Computer-aided design and optimization of a broad-band high frequency monolithic amplifier," IEEE J. Solid-State Circuits, vol. SC-7, pp. 487-492, Dec. 1972.

[14] S. Kakihana and P. H. Wang, "Simple CAD technique to develop high-frequency transistors," IEEE J. Solid-State Circuits, vol. SC-6, no. 4, pp. 236-243, Aug. 1971.

[15] J. Lange and W. N. Carr, "An application of device modeling to microwave power transistors," IEEE J. Solid-State Circuits, vol. SC-7, no. 1, pp. 71-80, Feb. 1972.

[16] M. K. Chen, "Methods for developing and assessing circuit models for bipolar diodes and transistors," Ph.D. dissertation, Univ. of Florida, Gainesville, 1989, chs. 4 and 9.

[17] A. B. Grebene, Bipolar and MOS Analog Integrated Circuit Design. New York: Wiley, 1984, pp. 220-223.

[18] W. F. Davis, "Bipolar design considerations for the automotive environment," IEEE J. Solid-State Circuits, vol. SC-8, pp. 419-426, Dec. 1973

[19] P. R. Motz and W. A. Vincent, "Automotive electronics: Designing custom ICs for a harsh environment," in Proc. IEEE Custom Integrated Circuits Conf, 1983, pp. 392-398.

\title{
CMOS OTA-C High-Frequency Sinusoidal Oscillators
}

\author{
Bernabé Linares-Barranco, Angel Rodríguez-Vázquez, \\ Edgar Sánchez-Sinencio, and José L. Huertas
}

\begin{abstract}
Several topology families are given to implement practical CMOS sinusoidal oscillators by using operational transconductance amplifier-capacitor (OTA-C) techniques. Design techniques are proposed taking into account the CMOS OTA's dominant nonidealities. Building blocks are presented for amplitude control, both by AGC schemes and by limitation schemes. Experimental results from 3- and $2-\mu \mathrm{m}$ CMOS (MOSIS) prototypes showing oscillation frequencies up to $69 \mathrm{MHz}$ are obtained. The amplitudes can be adjusted between $1 \mathrm{~V}$ peak to peak and $100 \mathrm{mV}$ peak to peak. Total harmonic distortions from $2.8 \%$ down to $0.2 \%$ have been experimentally measured in the laboratory.
\end{abstract}

\section{INTRODUCTION}

$\mathrm{T}$ HE USE OF circuits composed of operational transconductance amplifiers and capacitors (OTA-C's) has been

Manuscript received March 8, 1990; revised September 12, 1990. B. Linares-Barranco, A. Rodríguez-Vázquez, and J. L. Huertas were supported by the Spanish CICYT under Contract ME87-0004.

B. Linares-Barranco is with the Department of Electrical Engineering, Texas A\&M University, College Station, TX 77843 and the Depart mento de Diseño Analógico, Centro Nacional de Microelectrónica, Universidad de Sevilla, 41012 Sevilla, Spain.

A. Rodríguez-Vázquez and J. L. Huertas are with the Departmento de Diseño Analógico, Centro Nacional de Microelectrónica, Universidad de Sevilla, 41012 Sevilla, Spain.

E. Sánchez-Sinencio is with the Department of Electrical Engineering, Texas A\&M University, College Station, TX 77843.

IEEE Log Number 9041203. demonstrated to be potentially advantageous for the synthesis of high-frequency continuous-time monolithic analog operators, either linear [1]-[4], [11] or nonlinear [5]. One basic reason for the high-frequency potential of these circuits comes from the fact that the OTA is used in a local open loop. It means that no additional constraints are imposed on the frequency response due to local feedback-induced pole displacements [2]. Another advantage of open-loop OTAbased circuits is that the transconductance gain of the OTA is used as a design parameter. In a typical OTA architecture [12], this gain can be adjusted either by changing the tail current of a differential pair (fine adjustment) or by using digitally controlled current mirrors (coarse adjustment) [4]. Programmability is hence an inherent property of OTA-C circuits. Based upon the previous considerations, it may be expected that the transconductance amplifier-capacitor oscillators (TACO's) overcome the limitations in frequency and tunability of conventional op-amp-based $R C$-active oscillators. TACO's could then be applied for the design of highfrequency voltage-controlled sinusoidal oscillators (VCO's) with potential application in communication systems [6] and in the tuning of active filters [1]. In a companion paper [7] the authors have explored the synthesis of TACO's from classical oscillator models, namely quadrature and bandpassbased. The experimental results measured from discrete 


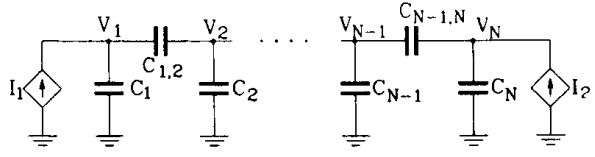

Fig. 1. General topology for the generation of second-order OTA-C oscillators.

bipolar prototypes showed good potential of the TACO's for high-frequency VCO's. Also, a 3- $\mu \mathrm{m}$ CMOS TACO including a limiting mechanism for controlling the amplitude has been reported [8] exhibiting a $10-\mathrm{MHz}$ frequency and THD down to $0.2 \%$. In this paper we first present a number of new architectures that can be systematically obtained from a general idealized TACO topology [9] and then provide experimental results for 2- and 3- $\mu \mathrm{m}$ CMOS prototypes up to 69 and $56 \mathrm{MHz}$, respectively. The results demonstrate that it is possible to implement high-frequency monolithic VCO oscillators based on simple OTA-C techniques and the modeling of the dominant OTA parasitic effects [3], [7]. Furthermore, we show that based on a general TACO structure, conventional and unconventional structures can be derived.

\section{OTA-C Oscillator Structures}

In this paper we are focusing on oscillators which can be ideally described by a second-order characteristic equation:

$$
s^{2}-b s+\Omega_{0}^{2}=0 .
$$

Fig. 1 shows a general topology for a second-order OTA-C oscillator structure [9]. The voltage-controlled current sources in this topology,

$$
I_{1}=\sum_{i=1}^{N} g_{1 i} V_{i} \quad I_{2}=\sum_{i=1}^{N} g_{2 i} V_{i}
$$

can be implemented by connecting OTA's in parallel, one per each different term in (2).

Parameters $b$ and $\Omega_{0}^{2}$ are given as functions of the OTA transconductance gains $g_{i j}$ and capacitor values. The basic TACO design goal is to achieve separate control of these former parameters with a minimum component count. We have systematically obtained different topologies from Fig. 1 to provide this feature. Some of the more interesting and practical ones are shown in Fig. 2. The corresponding expressions for $b$ and $\Omega_{0}^{2}$ are given in Table I. These structures involve a trade-off between complexity and degrees of freedom. At one end we will have structures with a minimum number of components but with a very limited degree of freedom. At the other, the structures will have larger component counts and more degrees of freedom. Nevertheless, we believe it is worthwhile to include the different structures since they are application dependent.

Ideally, for oscillation, the transconductance gains of Fig. 2 must be trimmed to yield $b=0$. However, in practical oscillators, due to the influence of parasitics, the poles are displaced from their nominal positions $\left(s_{p}= \pm j \Omega_{0}\right)$ to either the right or the left side of the complex frequency plane. For that reason, the oscillator must be designed to have its poles initially located inside the right-half complex frequency plane in order to assure self-starting operation, i.e., $b \geqslant \epsilon$, and $\epsilon$ is
TABLE I

IdeAL Expressions of $b$ AND $\Omega_{0}^{2}$ For the Different TACO

\begin{tabular}{|c|c|c|}
\hline$\left(g_{m 1}-g_{m 2}\right) C_{3}$ & $b_{c}$ & \multirow{2}{*}{ 它 } \\
\hline$\frac{g_{m 1} g_{m 2}}{\left(C_{1}+C_{3}\right)\left(C_{2}+C_{3}\right)-C_{3}^{2}}$ & $\Omega_{0 c}^{2}$ & \\
\hline$g_{m 3} C_{2}-g_{m 4} C_{1}$ & $b_{c}$ & \multirow{2}{*}{ 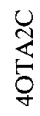 } \\
\hline$\frac{g_{m 1} g_{m 2}-g_{m 3} g_{m 4}}{C_{1} C_{2}}$ & $\Omega_{0 c}^{2}$ & \\
\hline$\left(g_{m 3}-g_{m 4}\right) C_{2}$ & $b_{c}$ & \multirow{2}{*}{ 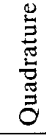 } \\
\hline$\frac{g_{m 1} g_{m 2}}{C_{1} C_{2}}$ & $\Omega_{0 c}^{2}$ & \\
\hline$\left(g_{m 3}-g_{m 4}\right) \frac{C_{1} C_{2} C_{3}}{\left(C_{1}+C_{3}\right)\left(C_{2}+C_{3}\right)}$ & $b_{c}$ & \multirow{3}{*}{$\frac{⿱ 乛 龰}{8}$} \\
\hline$g_{m 1} g_{m 2}\left(1+\frac{C_{1}}{C_{3}}\right)\left(1+\frac{C_{2}}{C_{3}}\right)-g_{m 3} g_{m 4} \frac{C_{1} C_{2}}{C_{3}^{2}}$ & \multirow{2}{*}{$\Omega_{0 c}^{2}$} & \\
\hline$C_{1} C_{2}$ & & \\
\hline
\end{tabular}
STRUCTURES

a slightly positive number [6]. Besides, nonlinearities have to be considered to explain the existence of stable oscillations. Using the natural nonlinear saturation characteristics of the OTA is the simplest form of limiter. Connecting a nonlinear resistor with a driving-point characteristic [7] is another approach providing better controllability. Finally, exploiting the bias terminals of the OTA's to implement an automatic gain control (AGC) mechanism is a more sophisticated scheme requiring additional circuitry but providing reduced harmonic distortion. These two latter alternatives, external limitation and AGC, have been used in the practical implementations included herein.

\section{INFLUENCE OF OTA PARASITICS}

In Table I we assume that the OTA performs as an ideal voltage-controlled current source. Some experimental errors can be expected as a consequence of using such an ideal model. For extreme frequencies (both high and low) the resulting errors are very large to be tolerated. Hence, for accurate TACO design at these extreme frequencies, OTA parasitics cannot be ignored in analyzing the proposed structures. Experimental observations [7], [10] reveal that only three parasitics have to be considered to obtain a valid design technique up to at least $69 \mathrm{MHz}$, as is demonstrated in the experimental results included in this paper:

a) output conductance $G_{o j}, 1 \leqslant j \leqslant 4$,

b) output and input capacitances, and

c) transconductance frequency dependency, $g_{m j} \rightarrow$ $g_{m j} \epsilon(s) \cong g_{m j}\left(1-s / \omega_{j}\right), 1 \leqslant j \leqslant 4$.

The following characteristic equation is obtained by using the describing function approach and considering the influence of parasitics:

$$
s^{2}-b_{c} s+\Omega_{0 c}^{2}=0
$$

where $b_{c}$ and $\Omega_{0 c}^{2}$ are functions of the transconductances $g_{m j}(1 \leqslant j \leqslant 4)$, capacitances $C_{i}(1 \leqslant i \leqslant 3)$, output conductances $G_{o j}(1 \leqslant j \leqslant 4)$, and parasitic zeros $\omega_{j}(1 \leqslant j \leqslant 4)$. 


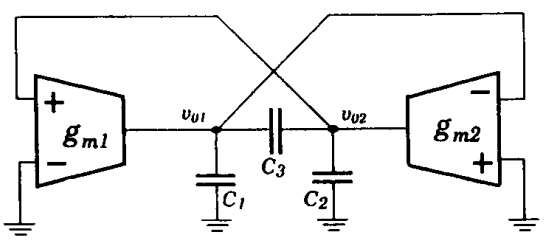

(a)



(b)

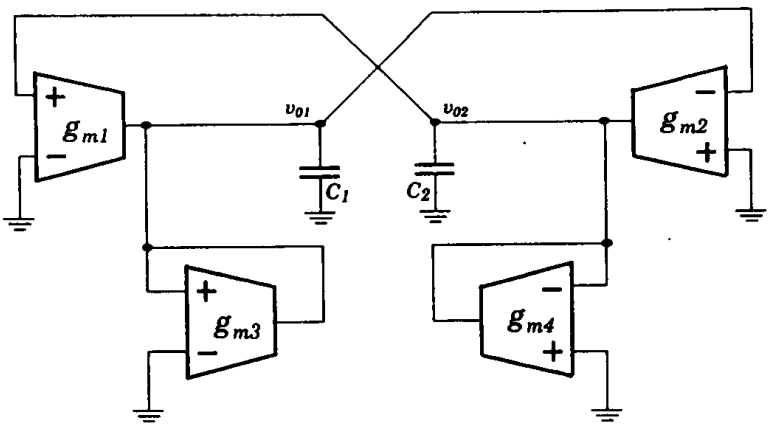

(c)

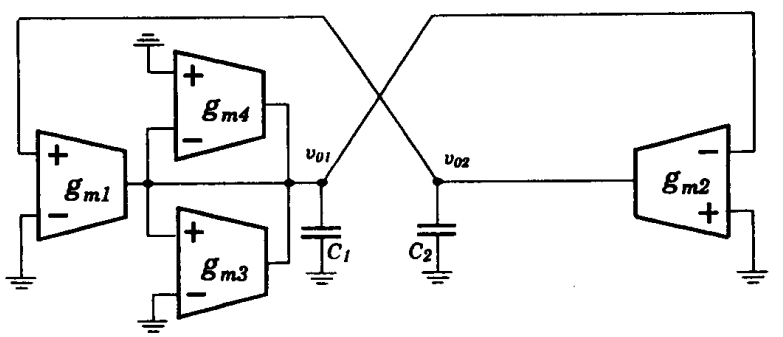

(d)

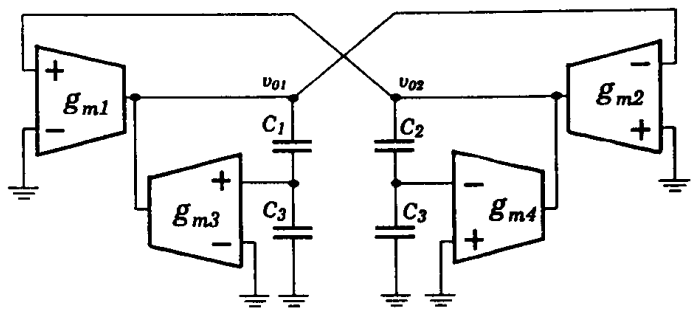

(e)

Fig. 2. OTA-C oscillator structures: (a) 2OTA3C, (b) 3OTA2C, (c) 4OTA2C, (d) quadrature, and (e) 4OTA4C.

Parasitics make the TACO oscillation condition $b_{c}$ and the oscillator frequency $\Omega_{0 c}^{2}$ depend on all the transconductance gains. It means that any intent to change $\Omega_{0 c}^{2}$ by any transconductance gain will also produce a change in $b_{c}$ and, hence, in the amplitude of the oscillations. For instance, in the 4OTA2C TACO, we can, ideally, change $\Omega_{0 c}^{2}$ via $g_{m 1}$ and $g_{m 2}$, without affecting $b$. However, when parasitics are taken into account, $g_{m 3}$ and $g_{m 4}$ must also be tuned to maintain $b_{c}$ constant. The influence of parasitics can be assessed from Fig. 3 corresponding to the 4OTA2C TACO. Fig. 3(a) shows trimming curves for the transconductance gains for the VCO operation and assuming the OTA's are ideal. Fig. 3(b) plots the corresponding curves in the case where parasitics are taken into account. Observe that one of the transconductance gains $g_{m 3}$ or $g_{m 4}$ can be made zero at any frequency. For low frequencies it is possible to make $g_{m 4}=0$ while at high frequencies $g_{m 3}=0$. At low frequencies, the output impedance (i.e., the OTA voltage gain) of the OTA's makes the oscillator deviate from the ideal (nonparasitic) behavior, while at high frequencies, it is the transconductance frequency dependence (excess phase) that produces the deviation. A way to avoid performance degradation due to parasitics and hence to yield high frequencies from the proposed TACO's is to use a predistortion technique based on the analysis of the parasitic's influence. We have used this method. The experimental results we have obtained confirm the validity of our approach.

\section{Experimental Results}

Three oscillator microchips were designed and fabricated in the CMOS p-well process, either $3-\mu \mathrm{m}$ double metal or $2-\mu \mathrm{m}$ double metal and double poly (through and thanks to MOSIS).

First Prototype: The prime objective of this first prototype, fabricated in the $3-\mu \mathrm{m}$ double-metal process, was to obtain a high enough oscillating frequency so that it could be considered a radio frequency. To fulfill this requirement, an OTA with a very high transconductance $g_{m}$ was needed. The OTA of Fig. 4 was designed for this purpose. Note that the architecture is a very simple one. The reason is that it can provide larger tuning ranges than linearized OTA's. On the other hand, since this OTA is going to be biased by a very large tail current (up to almost $10 \mathrm{~mA}$ ), there is no need for a linearization scheme. Table II shows the basic dc parameters of this OTA as a function of the bias voltage. The 


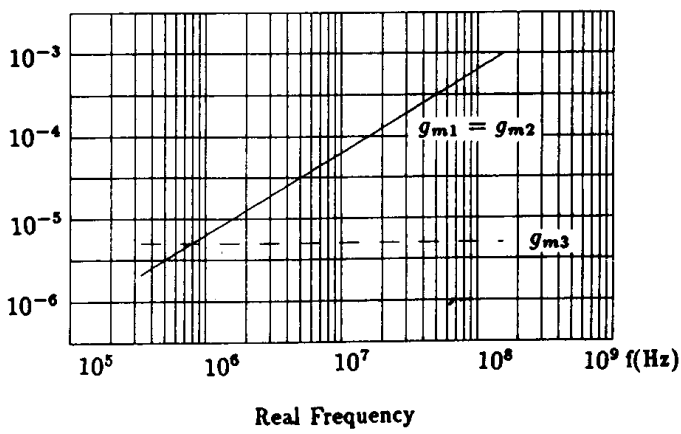

(a)

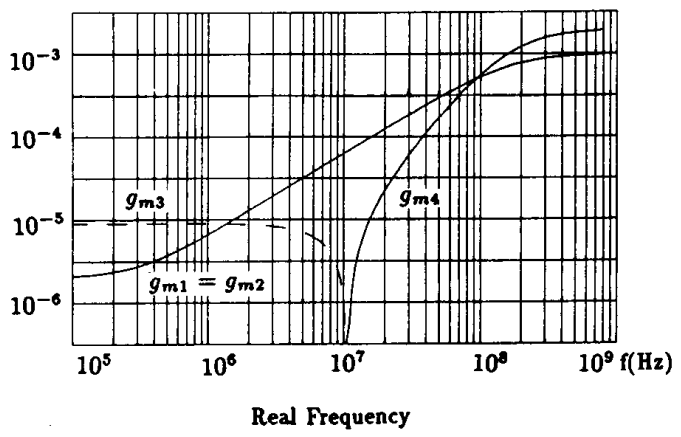

(b)

Fig. 3. Tuning of the $g_{m}$ 's for the VCO operation using the 4OTA2C: (a) without parasitics, and (b) with parasitics.

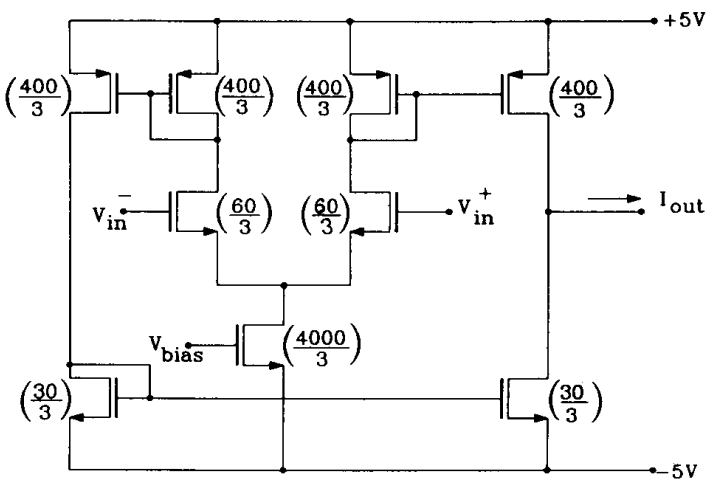

Fig. 4. A 3- - m CMOS OTA.

oscillator structure built was a quadrature oscillator (see Fig. $2(d)$ ), in which the OTA of transconductance $g_{m 3}$ was suppressed according to the predistortion technique that results from the OTA parasitic's influence, as is shown in Fig. 3(b). External limiters were included to control the amplitude. The frequency of the oscillator could be tuned between 12.0 and 56.1 MHz. The distortion measured at $56.1 \mathrm{MHz}$ was $2.5 \%$. In Table III the dependence of the oscillation frequency on the biasing (see $V_{\text {bias }}$ in Fig. 4) of the OTA's is shown. According to the parasitics' influence, the relation between oscillating frequency $\left(\Omega_{0}=2 \pi f_{0}\right)$, transconductance $g_{m i}$ of the different OTA's, capacitors $\left(C_{1}=C_{2}=\right.$ $5 \mathrm{pF}$ ), and parasitics (output impedances $G_{o i}$, and dominant
TABLE II

EXPERIMENTAL CHARACTERIZATION OF OTA

\begin{tabular}{c|c|c|c}
\hline$V_{\text {bias }}$ & \multicolumn{1}{|c|}{$R_{0}$} & $g_{m}$ & $I_{b}$ \\
\hline-2.72 & $2.77 \mathrm{k} \Omega$ & $2.49 \mathrm{mmhos}$ & $9.65 \mathrm{~mA}$ \\
-2.98 & $3.00 \mathrm{k} \Omega$ & $2.38 \mathrm{mmhos}$ & $9.60 \mathrm{~mA}$ \\
-3.19 & $3.78 \mathrm{k} \Omega$ & $2.12 \mathrm{mmhos}$ & $9.30 \mathrm{~mA}$ \\
-3.40 & $6.85 \mathrm{k} \Omega$ & $1.90 \mathrm{mmhos}$ & $6.10 \mathrm{~mA}$ \\
-3.51 & $9.47 \mathrm{k} \Omega$ & $1.76 \mathrm{mmhos}$ & $4.20 \mathrm{~mA}$ \\
-3.61 & $13.82 \mathrm{k} \Omega$ & $1.61 \mathrm{mmhos}$ & $2.70 \mathrm{~mA}$ \\
-3.68 & $18.91 \mathrm{k} \Omega$ & $1.43 \mathrm{mmhos}$ & $1.75 \mathrm{~mA}$ \\
-3.80 & $48.50 \mathrm{k} \Omega$ & $0.932 \mathrm{mmhos}$ & $780 \mu \mathrm{A}$ \\
-3.88 & $101.00 \mathrm{k} \Omega$ & 0.638 mmhos & $490 \mu \mathrm{A}$ \\
\hline
\end{tabular}

TABLE III

Experimental Characterization of Oscillator

\begin{tabular}{ccc}
\hline OTA1 OTA2 & OTA4 & Frequency \\
\hline$-2.9 \mathrm{~V}$ & $-3.39 \mathrm{~V}$ & $56.1 \mathrm{MHz}$ \\
$-3.19 \mathrm{~V}$ & $-3.35 \mathrm{~V}$ & $55.5 \mathrm{MHz}$ \\
$-3.40 \mathrm{~V}$ & $-3.35 \mathrm{~V}$ & $50.5 \mathrm{MHz}$ \\
$-3.51 \mathrm{~V}$ & $-3.37 \mathrm{~V}$ & $46.1 \mathrm{MHz}$ \\
$-3.61 \mathrm{~V}$ & $-3.41 \mathrm{~V}$ & $40.9 \mathrm{MHz}$ \\
$-3.68 \mathrm{~V}$ & $-3.51 \mathrm{~V}$ & $38.2 \mathrm{MHz}$ \\
$-3.80 \mathrm{~V}$ & $-3.66 \mathrm{~V}$ & $31.3 \mathrm{MHz}$ \\
$-3.88 \mathrm{~V}$ & $-3.80 \mathrm{~V}$ & $24.3 \mathrm{MHz}$ \\
$-3.96 \mathrm{~V}$ & $-3.84 \mathrm{~V}$ & $12.4 \mathrm{MHz}$ \\
$-3.98 \mathrm{~V}$ & $-3.86 \mathrm{~V}$ & $12.0 \mathrm{MHz}$ \\
\hline
\end{tabular}

parasitic pole $\omega_{i}$ of $g_{m i}$ ) is given for this oscillator structure by [10]

$$
\Omega_{0}^{2}=\frac{g_{m 1} g_{m 2}+G_{o 2}\left(g_{m 4}+G_{o 1}+G_{o 4}\right)}{C_{2}\left(C_{1}-\frac{g_{m 4}}{\omega_{4}}\right)+\frac{g_{m 1} g_{m 2}}{\omega_{1} \omega_{2}}} .
$$

To verify the accuracy of this expression, let us focus on Table III for the case of $24.3 \mathrm{MHz}$ of oscillating frequency. For this case, $g_{m 1}=g_{m 2}=0.64 \mathrm{mmhos}, g_{m 4}=0.93 \mathrm{mmhos}$, $G_{o 1}^{-1}=G_{o 2}^{-1}=101 \mathrm{k} \Omega, G_{o 4}^{-1}=48.5 \mathrm{k} \Omega, C_{1}=C_{2} \approx 5 \mathrm{pF}$, and $\omega_{1} \simeq \omega_{2} \simeq \omega_{4} \simeq 2 \pi \times 75 \mathrm{MHz}$. According to (4) this would yield a frequency of

$$
f_{0}=\frac{\Omega_{0}}{2 \pi}=26 \mathrm{MHz}
$$

which is very close to the $24.3 \mathrm{MHz}$ experimentally measured.

Second Prototype: A second microchip was fabricated in the $2-\mu \mathrm{m}$ double-poly, double-metal process in order to test the model oscillator structures proposed in this paper. The chip contains the three oscillators 2OTA3C, 4OTA2C, and 4OTA4C. This time a linearized OTA was used, as proposed by Nedungadi and Geiger [11]. The maximum bias current for the differential pair stage is less than $2 \mathrm{~mA}$. To obtain large $g_{m}$ values an additional current gain was added at the output current mirrors. In all these cases, the amplitude was controlled by limitation, using the CMOS nonlinear resistor of Fig. 5 [8], [10]. The maximum frequencies measured for the 2OTA3C, 4OTA2C, and 4OTA4C were $45.5,49.8$, and $69.0 \mathrm{MHz}$, respectively. Fig. 6 illustrates the variation of the frequency with the OTA bias voltage for each structure.

Third Prototype: A third microchip was designed in order to evaluate the performance of an OTA-C oscillator with AGC. A key component for the success of such a control loop is a peak detector. The oscillator will be made to operate between 3 and $13 \mathrm{MHz}$, approximately. In order for 


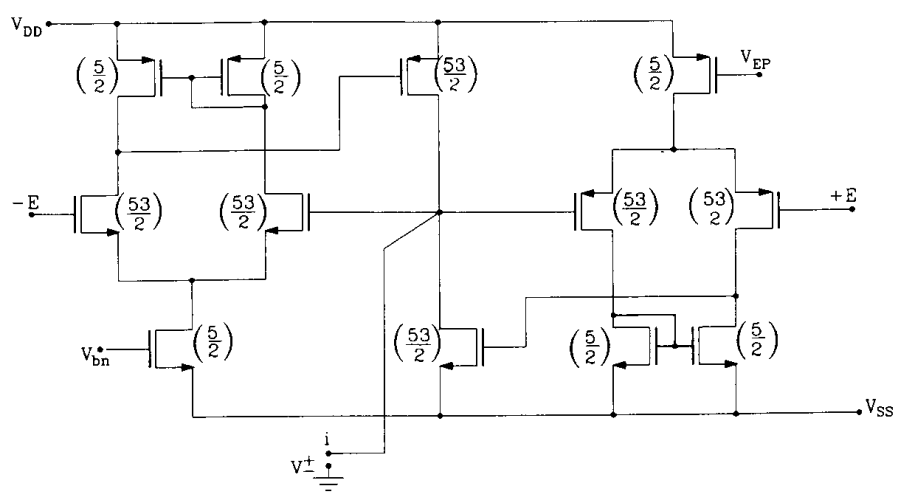

Fig. 5. CMOS implementation of the limiter.

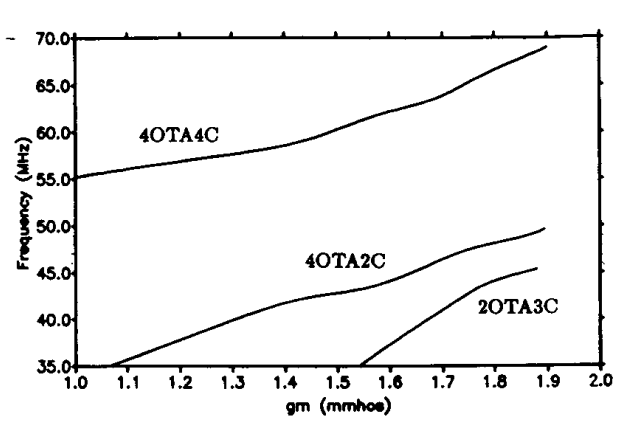

Fig. 6. Oscillation frequency versus OTA transconductances for 2OTA3C, 4OTA2C, and 4OTA4C oscillators.

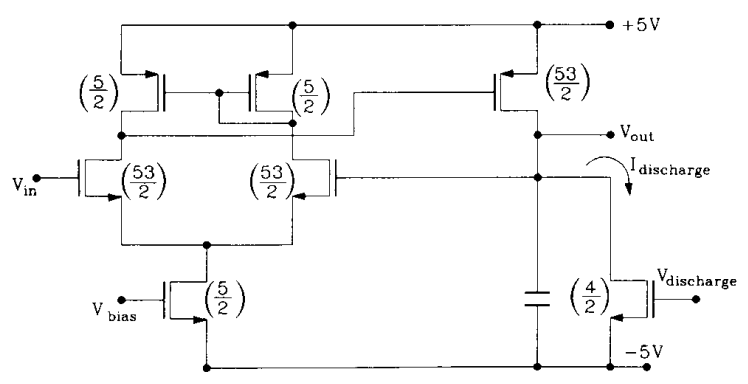

Fig. 7. CMOS peak detector.

the peak detector to cover this range and operate well at these frequencies a very simple circuit was chosen, as shown in Fig. 7. The performance of this peak detector was measured separately. By retuning the bias terminals $V_{\text {bias }}$ and $V_{\text {discharge }}$ (see Fig. 7), the peak detector was able to extract a 1-MHz signal from a $40-\mathrm{MHz}$ carrier, a $250-\mathrm{kHz}$ signal from a $1-\mathrm{MHz}$ carrier, or a $300-\mathrm{Hz}$ signal from a $10-\mathrm{kHz}$ carrier [10]. The oscillator structure used was a 4OTA2C, as shown in Fig. 8. The integrator and summer, added to the AGC loop in order to make it stable [10], are implemented using OTA-C techniques. The relationship between the oscillation frequencies and the bias voltage of OTA1 and OTA2 is shown in Fig. 9 for different values of the oscillation amplitude.

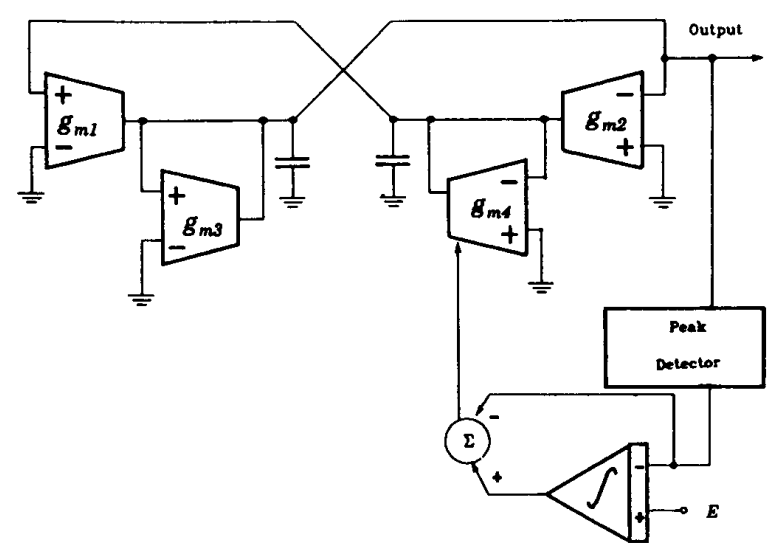

Fig. 8. 4OTA2C oscillator with AGC.

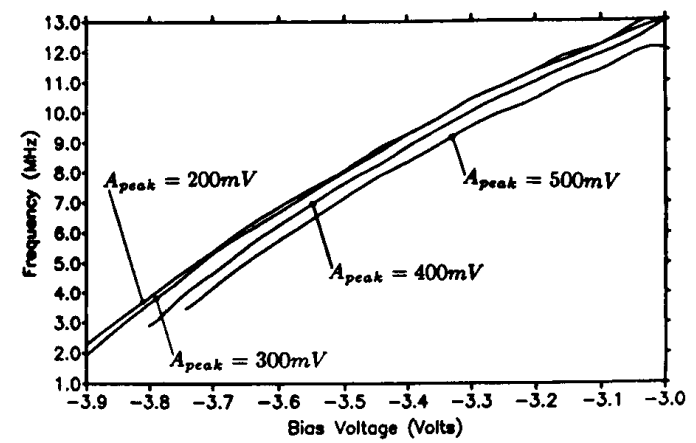

Fig. 9. Frequency versus bias voltage for different peak amplitudes.

\section{Conclusions}

A general approach for the systematic design of OTA-C oscillator structures is presented. Some novel oscillators are obtained and have been fabricated on silicon. Oscillation frequencies of up to $69 \mathrm{MHz}$ were measured. A wide-range simple peak detector has been fabricated and included in an AGC loop of one of the oscillators. The influence of the OTA's basic parasitics is discussed and verified in the opera- 
tion of the oscillators. In summary, the overall well-behaved performance of OTA-C oscillators for high frequencies has been demonstrated.

\section{REFERENCES}

[1] F. Krummenacher and N. Joel, "A 4 MHz CMOS continuoustime filter with on-chip automatic tuning," IEEE J. Solid-State Circuits, vol. 23, pp. 742-749, June 1988.

[2] K. D. Peterson, A. Nedungadi, and R. L. Geiger, "Amplifier design considerations for high frequency monolithic filters," in Proc. 1987 European Conf. Circuit Theory and Design, Sept. 1987, pp. 321-326.

[3] H. Nevárez-Lozano, J. A. Hill, and E. Sánchez-Sinencio, "Frequency limitations of continuous-time OTA-C filters," in Proc. IEEE / ISCAS '88, vol. 3 (Espoo, Finland), June 1988, pp. 2169-2172.

[4] K. H. Loh, D. Hiser, W. Adams, and R. L. Geiger, "A robust digitally programmable and reconfigurable monolithic filter structure," in Proc. 1989 IEEE Int. Symp. Circuits and Syst., May 1989, pp. 110-113.

[5] E. Sánchez-Sinencio, J. Ramírez-Angulo, B. Linares-Barranco, and A. Rodríguez-Vázquez, "Operational transconductance amplifier-based nonlinear function syntheses," IEEE J. SolidState Circuits, vol. 24, pp. 1576-1586, Dec. 1989.

[6] K. K. Clarke and D. T. Hess, Communication Circuits: Analysis and Design. Reading MA: Addison Wesley, 1978.

[7] A. Rodríguez-Vázquez, B. Linares-Barranco, J. L. Huertas, and E. Sánchez-Sinencio, "On the design of voltage controlled sinusoidal oscillators using OTA's," IEEE Trans. Circuits Syst., vol. 37, pp. 198-211, Feb. 1990.

[8] B. Linares-Barranco, A. Rodríguez-Vázquez, E. SánchezSinencio, and J. L. Huertas, "10 MHz CMOS OTA-C voltagecontrolled quadrature oscillator," Electron. Lett., vol. 25, pp. 765-766, June 1989.

[9] B. Linares-Barranco, A. Rodríguez-Vázquez, J. L. Huertas, E. Sánchez-Sinencio, and J. J. Hoyle, "Generation and design of sinusoidal oscillators using OTAs," in Proc. IEEE / ISCAS ' 88 , vol. 3 (Espoo, Finland), June 1988, pp. 2863-2866.

[10] B. Linares-Barranco, "Design of high frequency transconductance mode CMOS voltage controlled oscillators," Ph.D. dissertation, Univ. of Seville, Sevilla, Spain, May 1990 (available in English).

[11] A. Nedungadi and R. L. Geiger, "High-frequency voltage controlled continuous-time low-pass filter using linearized CMOS integrators," Electron. Lett., vol. 22, pp. 729-731, June 1986.

[12] P. E. Allen and D. R. Holberg, CMOS Analog Circuit Design. New York: Holt, Reinhart, Winston, 1987.

\title{
Two Novel Fully Complementary Self-Biased CMOS Differential Amplifiers
}

\author{
Mel Bazes
}

\begin{abstract}
Two novel CMOS differential amplifiers are presented. Both differ from conventional CMOS differential amplifiers in having fully complementary configurations and in being self-biased through negative feedback. The amplifiers have been applied as precision highspeed comparators in commercial VLSI CMOS integrated circuits.
\end{abstract}

\section{INTRODUCTION}

$\mathrm{T}$ HIS brief paper presents two novel CMOS differentia amplifiers. The first differential amplifier is intended for applications in which the input common-mode range is relatively limited; this amplifier is denoted a complementary self-biased differential amplifier (CSDA) [1]. The second differential amplifier is intended for applications in which the input common-mode range is bounded only by the supply voltages; this amplifier is denoted a very-wide-commonmode-range differential amplifier (VCDA) [2].

The circuit configurations of both amplifiers differ from those of conventional CMOS differential-amplifier configurations in two important ways:

1) the amplifiers are completely complementary, i.e., each n-type device operates in push-pull fashion with a corresponding p-type device;

Manuscript received April 11, 1990; revised September 5, 1990 The author is with Intel Israel, Ltd., 31015 Haifa, Israel. IEEE Log Number 9041476.
2) the amplifiers are self-biased through negative feedback.

These two differences in the amplifier configurations result in several performance enhancements:

- less sensitivity of active-region biasing to variations in processing, temperature, and supply;

- capability of supplying switching currents that are significantly greater than the quiescent bias current;

- nominal doubling of differential-mode gain $(+6 \mathrm{~dB})$.

These performance enhancements are particularly desirable in comparator applications in commercial digital CMOS VLSI integrated circuits, where precision, high speed, ease of interfacing to ordinary logic gates, and consistently high production yields are required. Both amplifiers have found application in commercial CMOS VLSI integrated circuits as precision comparators, as will be discussed below.

\section{CSDA}

\section{A. Theory of Operation}

A self-biased, but noncomplementary, CMOS differential amplifier has been reported [3], as has a fully complementary, but externally biased, CMOS differential amplifier [4]. 